\title{
Formação para a área da saúde: ensino, gestão, atenção e controle social
}

\section{Training for the health area: teaching, management, care and social control}

DOI: $10.46919 / \operatorname{archv1n5-002}$

Recebimento dos originais: 10/07/2020

Aceitação para publicação: 30/08/2020

\section{Maria Nauside Pessoa da Silva}

Graduada em Enfermagem; Teóloga

Mestra em Saúde da Família (UNINOVAFAPI)

Instituição: Faculdade Uninassau - Aliança: Rua Dr. Otto Tito, 278-306 - Redenção Teresina -PI (PIAUÍ/BRASIL)

Instituição: Faculdade de Educação do Piauí - FAEPI. Rua Treze de maio 2660 - Teresina - PI, (PIAUÍ/BRASIL)

Instituição: Faculdade Tecnológica de Teresina - CET. Rua Rio Grande do Norte, 790 - Pirajá, Teresina PI, (PIAUÍ/BRASIL)

E-mail: nauside@yahoo.com.br

\section{Antonio Marçal de Sousa Val}

Graduado em medicina pela Universidade Federal do Maranhão

Mestre em Saúde da Família (UNINOVAFAPI)

Instituição: Universidade Federal do Piauí -UFPI. Av. São Sebastião, no 2819 - Nossa Sra. de Fátima,

Parnaíba - PI (PIAUÍ/BRASIL)

E-mail: amasoval@hotmail.com

\section{Caroline Murad Abdalla}

Graduada em Fisioterapia

Mestra em Saúde da Família (UNINOVAFAPI)

Instituição: Centro Universitário - UNDB -Av. Colares Moreira, 443 - Jardim Renascença, São Luís MA (MARANHÃO/BRASIL)

E-mail: carolineabdalla@hotmail.com

\section{Gladys Carvalho de Araújo Alencar}

Graduada em Medicina

Mestra em Saúde da Família (UNINOVAFAPI)

Instituição: Universidade Estadual do Piauí - UESPI. Rua Olavo Bilac, 2335 - Centro (Sul), Teresina PI. (PIAUÍ/BRASIL)

E-mail: gladysalencar@yahoo.com.br

\section{Lorena Rocha Batista Carvalho}

Graduada em Enfermagem

Mestra em Saúde da Família (UNINOVAFAPI)

Instituição: Centro Universitário do Piauí - UNIFAPI. Rua Gov. Joca Píres, 1000 - Fátima, Teresina - PI (PIAUÍ/BRASIL)

E-mail: lorenarbcarvalho@gmail.com 


\section{Lennara de Siqueira Coêlho}

Graduada em Enfermagem

Mestra em Saúde da Família (UNINOVAFAPI)

Instituição: Centro Universitário do Piauí - UNIFAPI. Rua Gov. Joca Píres, 1000 - Fátima, Teresina - PI (PIAUÍ/BRASIL)

E-mail: lennara.coelho@hotmail.com

\section{Lília Maria Monteiro de Oliveira e Silva}

Graduada em Nutrição

Mestra em Saúde da Família (UNINOVAFAPI)

Instituição: Faculdade Estácio de Sá Unidade Teresina. Av. dos Expedicionários, 790 - São João,

Teresina - PI. (PIAUÍ/BRASIL)

E-mail: oliveira.lilia@estacio.br

\section{Marcelo de Moura Carvalho}

Graduado em Enfermagem

Doutor em Políticas de Saúde - Universidade Federal do Piauí - UFPI

Instituição: Fundação Municipal de Saúde - FMS. Rua Gov. Raimundo Artur Vasconcelos, 3015 -

Primavera, Teresina - PI. (PIAUÍ/BRASIL)

E-mail: Marcelo.mcarvalho@yahoo.com.br

\section{Teresa Marly Teles de Carvalho Melo Cirurgiã Dentista}

Mestra em Saúde da Família (UNINOVAFAPI)

Doutora em Engenharia Biomédica - UNIVAPI

Instituição: Instituto Federal do Piauí - IFPI. Rua Álvaro Mendes, 94 - Centro (Sul), Teresina - PI (PIAUÍ/BRASIL)

E-mail: hyramelo@yahoo.com.br

\section{RESUMO}

Estudo de revisão de literatura, que apresenta como objetivos: Realizar um estudo sobre o quadrilátero da formação para a área da saúde; Expressar sobre a significação dos processos de formação pelas necessidades sociais em saúde. $\mathrm{O}$ artigo tem como foco discorrer sobre o quadrilátero da formação para a área da saúde: "ensino" "gestão" "atenção" e "controle social". Pensando uma formação especializada para a prática em saúde visando a instituição e aplicabilidade das políticas de educação do (SUS) Sistema Único de Saúde. Conclui-se, que o desenho do quadrilátero da formação consolida uma construção organizada da formação para a saúde complementária da gestão do sistema de saúde, valorizando o controle social.

Palavras-chave: Formação, Educação permanente, Profissional, Saúde.

\footnotetext{
ABSTRACT

Literature review study, which aims to: Conduct a study on the training quadrilateral for the health area; Express about the significance of training processes for social health needs. The article focuses on talking about the quadrilateral of training for the health area: "teaching" "management" "attention" and "social control". Thinking of a specialized training for health practice aiming at the institution and applicability of the education policies of the (SUS) - Unified Health System. In conclusion, the design of the training quadrangle consolidates an organized construction of training for complementary health of the management of the health system, valuing social control.
} 
Keywords: Training, Permanent education, Professional, Cheers.

\section{INTRODUÇÃO}

O estudo aborda sobre uma nova lógica educativa para a formação dos profissionais da área da saúde, que transpõe a educação do domínio técnico - científico da profissão e segue pelos fundamentos de relações e de práticas que em conjunto contribuam para a melhoria da saúde da população, enfocando tanto o processo saúde - doença, quanto os fatores relacionados à gestão e estruturação do cuidado à saúde, onde enfoca a operacionalização do quadrilátero de formação para a área da saúde: ensino, gestão, atenção e controle social, sob a inclusão da educação permanente em saúde como constituinte de uma política pública nacional em ação. A lógica da educação permanente é descentralizadora, ascendente e transdisciplinar. O desenvolvimento da capacidade de aprendizagem, docência e de enfrentamento criativo das situações de saúde; de trabalhar em equipe e de melhorar permanentemente a qualidade do cuidado à saúde, bem como constituir práticas técnicas críticas, éticas e humanísticas.

\section{OBJETIVOS}

Realizar um estudo sobre o quadrilátero da formação para a área da saúde; expressar sobre a significação dos processos de formação pelas necessidades sociais em saúde.

\section{METODOLOGIA}

Estudo bibliográfico, utilizando - se o site do Ministério da Saúde e artigo publicado na Revista de Saúde Pública.

\section{RESULTADOS}

O controle social não se contrapõe à autonomia das instituições formadoras, uma vez que essa autonomia não pode implicar independência das políticas públicas e da regulação do Estado, ou interpor aos interesses públicos. A tarefa social de formar as novas gerações de profissionais, não deve estar atrelada à formação aos valores tradicionais, mas ao movimento de transformações na sociedade e sempre atender aos interesses coletivos e à construção de novidade em saberes e em práticas.

\section{DISCUSSÃO}

Considera-se que a formação de profissionais da área de saúde deve apontar para um trabalho de escuta, em que a interação entre profissional de saúde e usuário é determinante da qualidade da resposta assistencial, com responsabilidade técnico-científica, social e ética do cuidado, do tratamento ou do acompanhamento em saúde. O ‘quadrilátero' da ‘educação permanente em saúde é simples: análise e ação 
relativa simultaneamente à formação, à atenção, à gestão e à participação para que o trabalho em saúde seja lugar de atuação crítica, reflexiva, propositiva, compromissada e tecnicamente competente. Conforme aborda CECCIM; FEUERWERKERA (2004), a formação deve procurar ampliar às condições de atendimento às necessidades de saúde das pessoas individualmente e das populações de modo geral, também deve ampliar a visão da gestão setorial e do controle social em saúde, direcionando e apreciando o desenvolvimento da autonomia das pessoas considerando assim, a influência na formulação de políticas do cuidado. Portanto a atualização técnico-científica é um dos aspectos da qualificação das práticas desse modo a formação compreende também os aspectos de produção de subjetividade, produção de habilidades técnicas e de pensamento crítico reflexivo e o adequado conhecimento do Sistema Único de Saúde (SUS).

A presença de saberes clássicos das culturas ou a produção de formulações observadas atreladas ao processo saúde doença, cuidado e qualidade de vida pertence a lógicas distintas do modelo natural científico vigente entre os profissionais de saúde, isso tem se transformado em limitações à consolidação das metas de saúde, a gestão da formação como atividade meio, secundária à formulação de políticas de atenção à saúde (CECCIM, 2005).

\section{CONCLUSÃO}

Uma educação permanente sob a ótica do quadrilátero da formação para a área da saúde é de grande relevância para atuação dos profissionais da área, espera-se que essa política de formação realmente seja a diretriz para os atores que atuam na área da saúde e desejam realmente suprir as necessidades do SUS, objetivando a melhoria da saúde da população, assim, o quadrilátero da formação serve à construção e organização de uma gestão da educação na saúde integrante da gestão do sistema de saúde, reconstruindo a figura dos serviços como gestão e atenção em saúde e valorizando o controle social. Porém, a implementação efetiva do quadrilátero somente repercutirá na formação e na atenção quando todas as instâncias estiverem centralmente comprometidas com o ideal proposto. Recomenda-se o desenvolvimento de outros estudos acerca da temática em foco. 


\section{REFERÊNCIAS}

BRASIL. Ministério da Saúde. Conselho Nacional de Saúde. Princípios e diretrizes para NOB/RH-SUS. Brasília: Ministério da Saúde, 2003.

CECCIM, R. B; FEUERWERKER, L. C. M. O quadrilátero da formação para a área da saúde: ensino, gestão, atenção e controle social. Physis - Revista de Saúde Coletiva, 14(1): 41-66, 2004.

CECCIM, Ricardo Burg. Interface - Comunic, Saúde, Educ, v.9, n.16, p.161-77,set.2004/fev.2005 